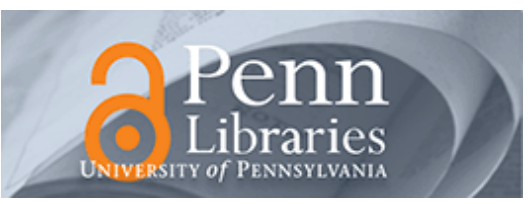

University of Pennsylvania

ScholarlyCommons

\title{
Nanoenabled microelectromechanical sensor for volatile organic chemical detection
}

\author{
Chiara Zuniga \\ University of Pennsylvania, zunigac@seas.upenn.edu \\ Matteo Rinaldi \\ University of Pennsylvania, rinaldim@seas.upenn.edu \\ Samuel M. Khamis \\ University of Pennsylvania, smk@physics.upenn.edu
}

A. T. Johnson
University of Pennsylvania, cjohnson@physics.upenn.edu

Gianbluça Piazzadidditional works at: https://repository.upenn.edu/ese_papers IIniversity of Pennsylvania, piazza@seas.upenn.edu

Part of the Acoustics, Dynamics, and Controls Commons, Biological Engineering Commons, Electrical and Electronics Commons, Electro-Mechanical Systems Commons, Electronic Devices and

Semiconductor Manufacturing Commons, Nanoscience and Nanotechnology Commons, and the Nanotechnology Fabrication Commons

\section{Recommended Citation}

Chiara Zuniga, Matteo Rinaldi, Samuel M. Khamis, A. T. Johnson, and Gianluca Piazza, "Nanoenabled microelectromechanical sensor for volatile organic chemical detection", . June 2009.

Copyright 2009 American Institute of Physics. This article may be downloaded for personal use only. Any other use requires prior permission of the author and the American Institute of Physics. Reprinted from:

Nanoenabled microelectromechanical sensor for volatile organic chemical detection Chiara Zuniga, Matteo Rinaldi, Samuel M. Khamis, A. T. Johnson, and Gianluca Piazza, Appl. Phys. Lett. 94, 223122 (2009), DOI:10.1063/

1.3151919 URL: http://link.aip.org/link/?APPLAB/94/223122/1

This paper is posted at ScholarlyCommons. https://repository.upenn.edu/ese_papers/494

For more information, please contact repository@pobox.upenn.edu. 


\title{
Nanoenabled microelectromechanical sensor for volatile organic chemical detection
}

\author{
Abstract \\ A nanoenabled gravimetric chemical sensor prototype based on the large scale integration of single- \\ stranded DNA (ss-DNA) decorated single-walled carbon nanotubes (SWNTs) as nanofunctionalization \\ layer for aluminum nitride contour-mode resonant microelectromechanical (MEM) gravimetric sensors \\ has been demonstrated. The capability of two distinct single strands of DNA bound to SWNTs to enhance \\ differently the adsorption of volatile organic compounds such as dinitroluene (simulant for explosive \\ vapor) and dymethyl-methylphosphonate (simulant for nerve agent sarin) has been verified \\ experimentally. Different levels of sensitivity $\left(17.3\right.$ and $\left.28 \mathrm{KHz} \mu \mathrm{m}^{\wedge} 2 / \mathrm{fg}\right)$ due to separate frequencies of \\ operation ( 287 and $450 \mathrm{MHz}$ ) on the same die have also been shown to prove the large dynamic range of \\ sensitivity attainable with the sensor. The adsorption process in the ss-DNA decorated SWNTs does not \\ occur in the bulk of the material, but solely involves the surface, which permits to achieve $50 \%$ recovery in \\ less than $29 \mathrm{~s}$.

\section{Keywords} \\ MEMS, microsensors, chemical sensors, carbon nanotubes, DNA, selectivity

\section{Disciplines} \\ Acoustics, Dynamics, and Controls | Biological Engineering | Electrical and Electronics | Electro- \\ Mechanical Systems | Electronic Devices and Semiconductor Manufacturing | Nanoscience and \\ Nanotechnology | Nanotechnology Fabrication

\section{Comments} \\ Copyright 2009 American Institute of Physics. This article may be downloaded for personal use only. Any \\ other use requires prior permission of the author and the American Institute of Physics. Reprinted from: \\ Nanoenabled microelectromechanical sensor for volatile organic chemical detection Chiara Zuniga, \\ Matteo Rinaldi, Samuel M. Khamis, A. T. Johnson, and Gianluca Piazza, Appl. Phys. Lett. 94, 223122 \\ (2009), DOI:10.1063/1.3151919 URL: http://link.aip.org/link/?APPLAB/94/223122/1
}




\title{
Nanoenabled microelectromechanical sensor for volatile organic chemical detection
}

\author{
Chiara Zuniga, ${ }^{1, a)}$ Matteo Rinaldi, ${ }^{1, a)}$ Samuel M. Khamis, ${ }^{2}$ A. T. Johnson, ${ }^{2}$ and \\ Gianluca Piazza ${ }^{1}$ \\ ${ }^{1}$ Department of Electrical and Systems Engineering, University of Pennsylvania, Philadelphia, \\ Pennsylvania 19104, USA \\ ${ }^{2}$ Department of Physics and Astronomy, University of Pennsylvania, Philadelphia, Pennsylvania \\ 19104, USA
}

(Received 26 February 2009; accepted 17 May 2009; published online 5 June 2009)

\begin{abstract}
A nanoenabled gravimetric chemical sensor prototype based on the large scale integration of single-stranded DNA (ss-DNA) decorated single-walled carbon nanotubes (SWNTs) as nanofunctionalization layer for aluminum nitride contour-mode resonant microelectromechanical (MEM) gravimetric sensors has been demonstrated. The capability of two distinct single strands of DNA bound to SWNTs to enhance differently the adsorption of volatile organic compounds such as dinitroluene (simulant for explosive vapor) and dymethyl-methylphosphonate (simulant for nerve agent sarin) has been verified experimentally. Different levels of sensitivity (17.3 and $28 \mathrm{KHz} \mu \mathrm{m}^{2} / \mathrm{fg}$ ) due to separate frequencies of operation $(287$ and $450 \mathrm{MHz}$ ) on the same die have also been shown to prove the large dynamic range of sensitivity attainable with the sensor. The adsorption process in the ss-DNA decorated SWNTs does not occur in the bulk of the material, but solely involves the surface, which permits to achieve 50\% recovery in less than 29 s. (c) 2009 American Institute of Physics. [DOI: 10.1063/1.3151919]
\end{abstract}

In recent years, much effort has been dedicated to the fabrication of micromachined resonant devices for high performance chemical sensing. Gravimetric sensors, based on a MEMS/NEMS (Micro/Nanoelectromechanical System) resonator and an integrated adsorbent layer that binds analyte molecules, offer the advantage of passive, wireless, and remote readout. Crucial parameters for such volatile organic chemical (VOC) detectors include sensitivity, selectivity, and response times, which depend on the resonance frequency of the device and the properties of its chemically active sensitive layer. Size, portability, ease of monitoring, and cost are other important considerations for practical environmental sensors.

Resonant MEMS/NEMS devices, such as cantilever beams ${ }^{1}$ and thin film bulk acoustic resonators (FBARs), ${ }^{2}$ have shown potential as gravimetric sensors. Their reduced size results in higher frequencies of operation than conventional quartz crystal devices ${ }^{3}$ and unprecedented values of mass sensitivity. However, improved mass sensitivity may not translate into improved analyte concentration sensitivity, because the reduced size of a MEMS/NEMS resonator also limits the area dedicated to the adsorbent layer, and thus the mass adsorption in the presence of a given analyte concentration. The implementation of a high performance gravimetric sensor array for detection of multiple VOCs also requires an excellent chemically active adsorbent layer. In this letter, we demonstrate high performance gravimetric sensors for VOC detection that uses single-stranded DNA (ss-DNA) decorated single-walled carbon nanotubes (SWNTs) as nanofunctionalization layer on top of aluminum nitride (AlN) contour-mode MEMS resonators (CMRs) (Ref. 4) (Fig. 1). The AlN MEMS resonator offers key advantages over previ-

\footnotetext{
a) Authors to whom correspondence should be addressed. Electronic addresses: zunigac@seas.upenn.edu and rinaldim@seas.upenn.edu.
}

ously demonstrated MEMS/NEMS resonator technologies because of the high frequency of operation (sensitivity scales with frequency squared), the high quality factor in air $(1000-3000),{ }^{4}$ the large area available for sensing and especially the ability to provide for multiple frequencies on the same silicon substrate..$^{5}$ The device center frequency is in fact defined lithographically, which permits to attain different frequencies of operation (10 $\mathrm{MHz}$ to $3 \mathrm{GHz}$ ) and consequently enhance the dynamic range (defined as ratio of maximum to minimum concentration of detectable analyte) of the sensor platform in which it will be employed.

Regarding the adsorbent layer, SWNTs have shown great potential for gas sensing and have been employed as both chemically active sensitive material and transducers. ${ }^{6}$ In particular, SWNTs offer advantages as an adsorbent layer compared to polymeric films. Thanks to their extremely large surface to volume ratio, SWNTs provide the resonator with a large number of adsorption sites distributed along only two dimensions, therefore enabling a quick and reversible surface adsorption process. This cannot be achieved with polymeric films for which the adsorption process occurs within the bulk of the film. At the same time, SWNTs offers a better acoustic impedance match to the resonator than polymers, and because of their reduced thickness, they limit any impact on the resonator performance (such as quality factor and electromechanical coupling). Nevertheless, the use of bare carbon nanotubes as nanosensitive layer is not sufficient to address the problem of selectivity, since SWNTs are very sensitive to a broad target of molecules. In this work, in order to overcome the lack of specificity intrinsic to pristine SWNTs, a nanofunctionalizing biocoating constituted by ss-DNAdecorated SWNTs has been demonstrated. Although, DNAdecorated SWNTs have been employed for the fabrication of chemically sensitive FET devices ${ }^{7}$ as well as fluorescence based sensors ${ }^{8}$ their physical and chemical properties have 


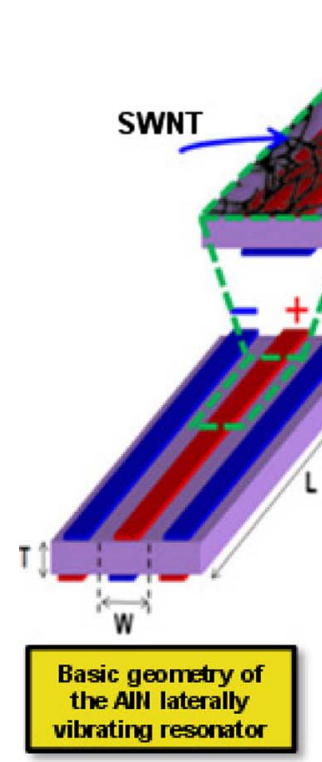

(a)

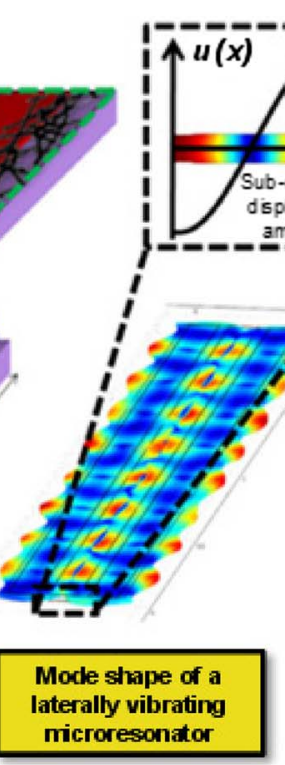

(b)
Zoo med view of SWNT mat

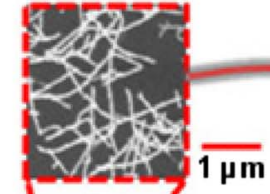
$1 \mu \mathrm{m}$

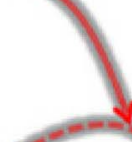

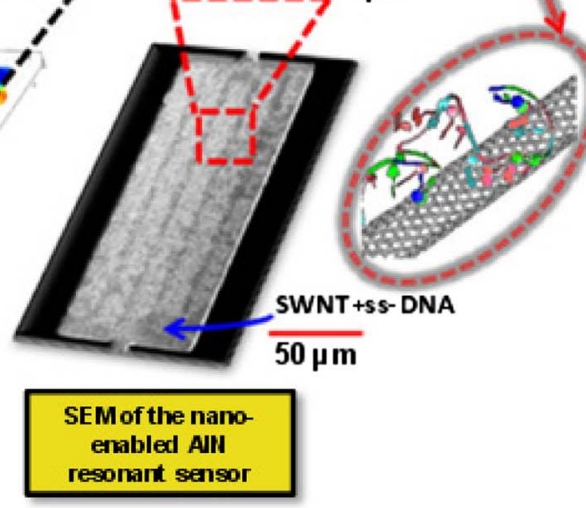

(c) never been investigated for gravimetric sensors.

In this work, high purity SWNT were first grown by chemical vapor deposition (CVD) directly on top of two AlN contour-mode resonators fabricated on the same chip and operating at two different frequencies (287 and $450 \mathrm{MHz}$ ). Then, the two devices were functionalized with two distinct sequences of ss-DNA and exposed to gas species including 2,6 dinitroluene (DNT), an explosive vapor, and dymethylmethylphosphonate (DMMP), a simulant for nerve agent sarin, demonstrating up to an eightfold enhancement in DNT detection using one sequence of ss-DNA and a two to three times increase in both species detection when a second sequence was used. This constitutes the experimental verification that two distinct strands of DNA respond differently to separate analytes when employed for monitoring mass adsorption.

As previously reported, ${ }^{5}$ the effective sensitivity $\left(S_{\text {eff }}\right)$ to adsorbed mass per unit area for a contour-mode resonator can be expressed as in

$$
S_{\text {eff }}=-\frac{f_{0}^{2}}{\sqrt{E_{0} \rho_{0}}} \frac{W}{T},
$$

where $f_{0}$ is the operating frequency of the resonator, while $W$ and $T$ are the geometric dimensions of the device as represented in Fig. 1(a).

The effective sensitivity value for the fabricated devices was analytically evaluated and found to be $17.3 \mathrm{KHz}$ $\mu \mathrm{m}^{2} /$ fg for a $287 \mathrm{MHz}$ resonator $(T=2 \mu \mathrm{m}, L=200 \mu \mathrm{m}$, $W=15 \mu \mathrm{m}$, and $n=5)$ and $28 \mathrm{KHz} \mu \mathrm{m}^{2} /$ fg for a $442 \mathrm{MHz}$ resonator $(T=2 \mu \mathrm{m}, L=200 \mu \mathrm{m}, W=10 \mu \mathrm{m}$, and $n=5)$, which are up to four times better than the ones achievable with FBAR devices operating in the same frequency range. ${ }^{2}$ A 2D finite element method (FEM) analysis using COMSOL MULTIPHYSICS confirmed these analytical values.

A combination of top-down microfabrication techniques for the resonators and bottom-up growth for SWNTs was employed to realize the nanoenabled microelectromechanical sensors of this work. The resonant AlN devices were fabricated using a process based on what was previously demonstrated by Piazza et al. ${ }^{9}$ A catalytic CVD nanotube growth process $^{7}$ was performed immediately before the resonator was released from the $\mathrm{Si}$ substrate. Thanks to the unique set of materials used to manufacture the resonators, the CVD process could occur at temperatures around $900{ }^{\circ} \mathrm{C}$.

This fabrication process permits the large scale integration of bottom-up grown nanotubes with top-down fabricated resonators. Although this process was demonstrated at the die level because the CVD reactor cannot accommodate bigger substrates, it can be effectively extended to wafer level ${ }^{10}$ and it is therefore amenable to large scale manufacturing processes. The ss-DNA functionalization of the SWNT layer is the last step of the process and it was performed after the resonator was released from the silicon substrate. Each DNA sequence (obtained from Invitrogen) was first diluted in distilled water to make a solution of $100 \mu \mathrm{M}$. The solution was applied on the specific device with a micropipette and let rest for $45 \mathrm{~min}$ in a humid environment. Hence it was dried in a nitrogen stream. The ss-DNA sequences chosen as functionalizing agents ${ }^{7,8}$ were

\section{sequence 1: 5' GAC TCT GTG GAG GAG GTA GTC 3' sequence 2: 5' CTT CTG TCT TGA TGT TTG TCA AAC $3^{\prime}$}

and were applied, respectively, to the 450 and $287 \mathrm{MHz}$ resonators. It is worth noting that the described process permits to functionalize adjacent transducers on the same chip with different chemical coatings, therefore enabling the realization of on-chip electronic noses. The fabricated nanoenabled resonators were exposed to varying concentrations of DNT and DMMP $(10 \%, 25 \%$, and $50 \%$ of their saturated vapor pressure) before and after the ss-DNA functionalization of the SWNTs adsorbent layer (the measurement setup is described elsewhere ${ }^{5}$ ).

The response of the $450 \mathrm{MHz}$ resonator functionalized with sequence 1 demonstrated up to eightfold enhancement in the adsorption of DNT with respect to bare SWNTs [Fig. 2(a)] and only a factor of two to three enhancements for DMMP [Fig. 2(b)]. On the contrary, no substantial diversification in the response to DNT and DMMP was recorded for the $287 \mathrm{MHz}$ resonator functionalized with sequence 2. In 


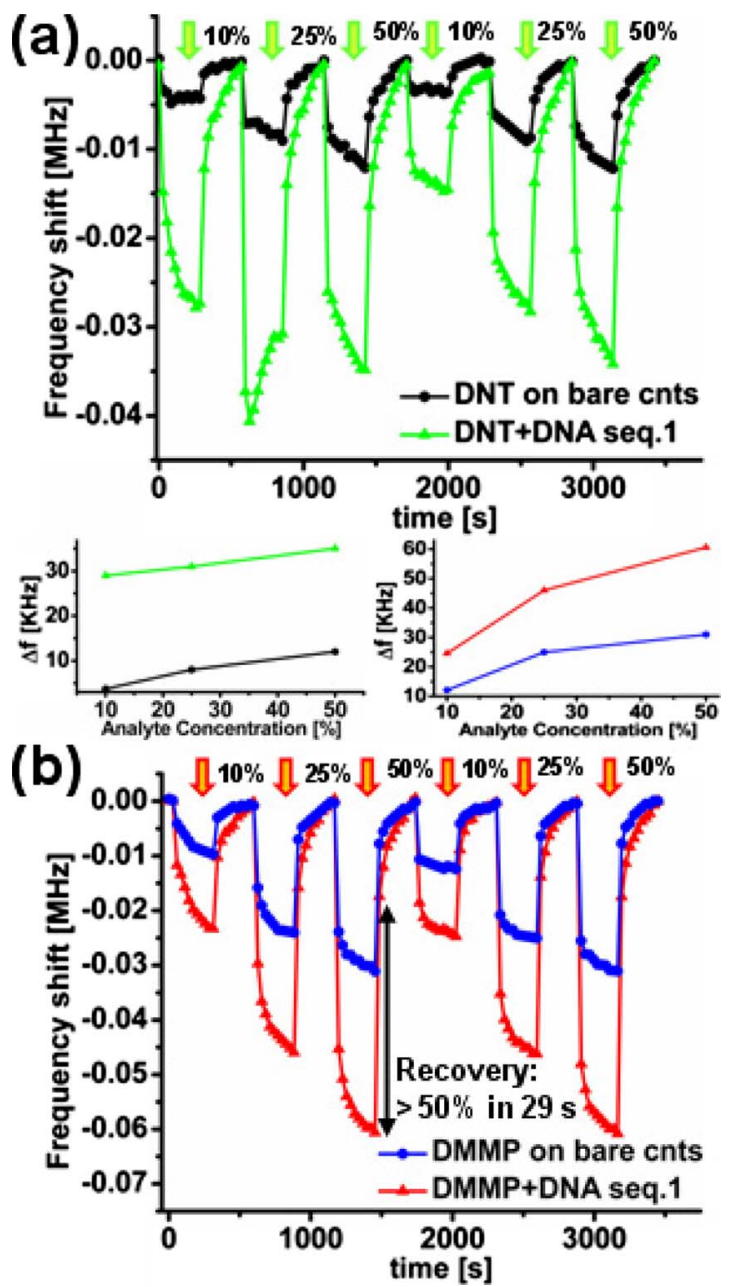

FIG. 2. (Color online) Response to DNT (a) and DMMP (b) of a $450 \mathrm{MHz}$ AlN contour-mode resonator with and without DNA seq. 1 functionalization. The results prove that the DNA sequence1 enhances the device sensitivity. The response to $10 \%$ and $25 \%$ DNT shows different patterns depending on the number of injections. This result is repeatable, but its physics is still under investigation. The two insets show the measured frequency shift as a function of the analyte concentration.

fact, this device exhibited an improvement in the response to both chemical agents and both species adsorption was amplified by a factor of nearly two to three folds over the bare SWNT-coated resonator.

The estimated VOC mass adsorbed for $10 \%$ concentration of both analytes is reported in Fig. 3, where the diversification in the affinity to different chemical species clearly points out that the adsorption process is DNA sequence dependent. For both sensors $50 \%$ recovery was measured in approximately $29 \mathrm{~s}$ by flowing solely Ar, therefore proving that the sensor is reversible.

The predicted values of sensitivity for the fabricated devices $\left(17.3 \mathrm{KHz} \mu \mathrm{m}^{2} / \mathrm{fg}\right.$ for a $287 \mathrm{MHz}$ resonator and $28 \mathrm{KHz} \mu \mathrm{m}^{2} /$ fg for a $450 \mathrm{MHz}$ resonator) were experimentally verified by fitting the electrical response of the resonator to the modified Butterworth van Dyke (MBVD) model. ${ }^{11}$ After the application of the ss-DNA, a decrease in the resonator quality factor $Q$ of only $10 \%$ was recorded for both devices showing that the $Q$ of the resonant sensors presented

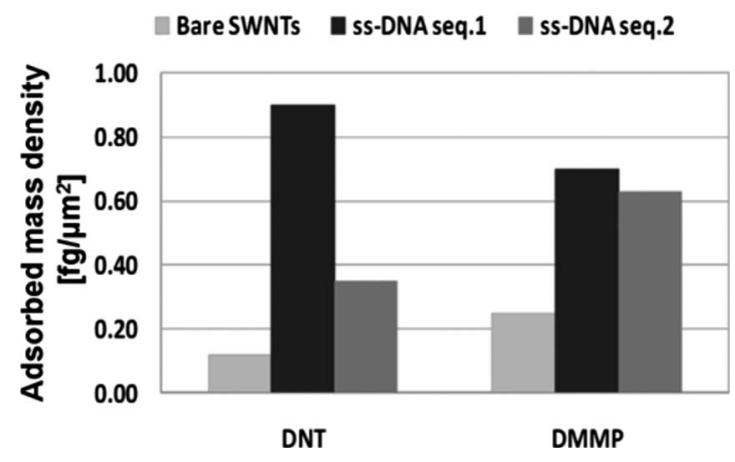

FIG. 3. Comparison between DNT and DMMP response with different ssDNA functionalization sequences for $10 \%$ analyte concentration. The chart shows the enhanced mass adsorption occurred because of the functionalization process and the ability to selectively distinguish between DNT and DMMP.

here is not significantly altered by the nanofunctionalization layer.

To summarize, design, fabrication, and testing of a gravimetric sensor prototype based on the large scale integration of ss-DNA decorated SWNTs on AlN contour-mode resonator have been demonstrated. The nanofunctionalization layer permits to achieve up to an eightfold increase in sensitivity to DNT over bare nanotubes with one sequence of DNA, and a two to three fold enhancement to both DNT and DMMP when a second sequence is employed. Two different levels of sensitivity equal to 17.3 and $28 \mathrm{KHz} \mu \mathrm{m}^{2} /$ fg were measured on the same silicon chip, therefore providing a proofof-concept of the high dynamic sensing range attainable by these devices. This work constitutes the initial experimental verification toward the demonstration of a highly selective nanomechanical nose, whose realization will be made possible by gaining access to a vast ss-DNA library.

The first two authors, C.Z. and M.R., contributed equally to this work. This work was supported by NCMR and NSF/ DIA (Grant No. IIS-07-15024). The authors wish to thank the staff at the University of Pennsylvania Wolf Nanofabrication Facility (WNF).

${ }^{1}$ M. Li, H. X. Tang, and M. L. Roukes, Nat. Nanotechnol. 2, 114 (2007).

${ }^{2}$ M. Penza, P. Aversa, G. Cassano, D. Suriano, W. Wlodarski, M. Benetti, D. Cannata, F. Di Pietrantonio, and E. Verona, IEEE Trans. Electron Device 55, 1237 (2008).

${ }^{3}$ D. S. Ballantine, R. M. White, S. J. Martin, A. J. Ricco, E. T. Zellers, G. C. Frye, and H. Wohltjen, Acoustic Wave Sensors Theory, Design and Physco-Chemical Applications (Academic, San Diego, 1997).

${ }^{4}$ G. Piazza, P. J. Stephanou, and A. P. Pisano, Solid State Electron. 51, 1596 (2007).

${ }^{5}$ M. Rinaldi, C. Zuniga, N. Sinha, M. Taheri, S. Khamis, A. T. Johnson, and G. Piazza, Proceedings of the IEEE Frequency Control Symposium, 2008, pp. 443-448.

${ }^{6}$ T. Zhang, S. Mubeen, N. V. Myung, and M. A. Deshusses, Nanotechnology 19, 332001 (2008).

${ }^{7}$ C. Staii, A. T. Johnson, M. Chen, and A. Gelperin, Nano Lett. 5, 1774 (2005).

${ }^{8}$ J. White, K. Truesdell, L. B. Williams, M. S. AtKisson, and J. S. Kauer, PLoS Biol. 6, (2008).

${ }^{9}$ G. Piazza, P. J. Stephanou, and A. P. Pisano, J. Microelectromech. Syst. 16, 319 (2007).

${ }^{10}$ N. R. Franklin, Y. Li, R. J. Chen, A. Javey, and H. Daia Appl. Phys. Lett. 79, 4571 (2001).

${ }^{11}$ G. Piazza, P. J. Stephanou, and A. P. Pisano, J. Microelectromech. Syst. 15, 1406 (2006). 